\title{
ON UNIVALENT FUNCTIONS DEFINED BY A GENERALIZED SĂLĂGEAN OPERATOR
}

\author{
F. M. AL-OBOUDI
}

Received 17 August 2001 and in revised form 18 March 2002

We introduce a class of univalent functions $R^{n}(\lambda, \alpha)$ defined by a new differential operator $D^{n} f(z), n \in \mathbb{N}_{0}=\{0,1,2, \ldots\}$, where $D^{0} f(z)=f(z), D^{1} f(z)=(1-\lambda) f(z)+\lambda z f^{\prime}(z)=$ $D_{\lambda} f(z), \lambda \geq 0$, and $D^{n} f(z)=D_{\lambda}\left(D^{n-1} f(z)\right)$. Inclusion relations, extreme points of $R^{n}(\lambda, \alpha)$, some convolution properties of functions belonging to $R^{n}(\lambda, \alpha)$, and other results are given.

2000 Mathematics Subject Classification: 30C45.

1. Introduction. Let $A$ denote the class of functions of the form

$$
f(z)=z+\sum_{k=2}^{\infty} a_{k} z^{k},
$$

analytic in the unit disc $\Delta=\{z:|z|<1\}$.

We denote by $R(\alpha)$ the subclass of $A$ for which $\operatorname{Re} f^{\prime}(z)>\alpha$ in $\Delta$. For a function $f$ in $A$, we define the following differential operator:

$$
\begin{gathered}
D^{0} f(z)=f(z), \\
D^{1} f(z)=(1-\lambda) f(z)+\lambda z f^{\prime}(z)=D_{\lambda} f(z), \quad \lambda \geqslant 0, \\
D^{n} f(z)=D_{\lambda}\left(D^{n-1} f(z)\right) .
\end{gathered}
$$

If $f$ is given by (1.1), then from (1.3) and (1.4) we see that

$$
D^{n} f(z)=z+\sum_{k=2}^{\infty}[1+(k-1) \lambda]^{n} a_{k} z^{k} .
$$

When $\lambda=1$, we get Sălăgean's differential operator [8].

Let $R^{n}(\lambda, \alpha)$ denote the class of functions $f \in A$ which satisfy the condition

$$
\operatorname{Re}\left(D^{n} f(z)\right)^{\prime}>\alpha, \quad z \in \Delta,
$$

for some $0 \leq \alpha \leq 1, \lambda \geqslant 0$, and $n \in \mathbb{N}_{0}=\{0,1,2, \ldots\}$. It is clear that $R^{0}(\lambda, \alpha) \equiv R(\alpha) \equiv$ $R^{n}(0, \alpha)$ and that $R^{1}(\lambda, \alpha) \equiv R(\lambda, \alpha)$, the class of functions $f \in A$ satisfying

$$
\operatorname{Re}\left(f^{\prime}(z)+\lambda z f^{\prime \prime}(z)\right)>\alpha, \quad z \in \Delta
$$

studied by Ponnusamy [5] and others. 
The Hadamard product or convolution of two power series $f(z)=\sum_{k=0}^{\infty} a_{k} z^{k}$ and $g(z)=\sum_{k=0}^{\infty} b_{k} z^{k}$ is defined as the power series $(f * g)(z)=\sum_{k=0}^{\infty} a_{k} b_{k} z^{k}, z \in \Delta$.

The object of this paper is to derive several interesting properties of the class $R^{n}(\lambda, \alpha)$ such as inclusion relations, extreme points, some convolution properties, and other results.

2. Inclusion relations. Theorem 2.3 shows that the functions in $R^{n}(\lambda, \alpha)$ belong to $R(\alpha)$ and hence are univalent. We need the following lemmas.

LEMMA 2.1. If $p(z)$ is analytic in $\Delta, p(0)=1$ and $\operatorname{Re} p(z)>1 / 2, z \in \Delta$, then for any function $F$ analytic in $\Delta$, the function $p * F$ takes its values in the convex hull of $F(\Delta)$.

The assertion of Lemma 2.1 follows by using the Herglotz representation for $p$. The next lemma is due to Fejér [3].

A sequence $a_{0}, a_{1}, \ldots, a_{n}, \ldots$ of nonnegative numbers is called a convex null sequence if $a_{n} \rightarrow 0$ as $n \rightarrow \infty$ and

$$
a_{0}-a_{1} \geqslant a_{1}-a_{2} \geqslant \cdots \geqslant a_{n}-a_{n+1} \geqslant \cdots \geqslant 0 .
$$

LEMMA 2.2. Let $\left\{c_{k}\right\}_{k=0}^{\infty}$ be a convex null sequence. Then the function $p(z)=c_{0} / 2+$ $\sum_{k=1}^{\infty} c_{k} z^{k}, z \in \Delta$, is analytic and $\operatorname{Re} p(z)>0$ in $\Delta$.

Now we prove the following theorem.

THEOREM 2.3.

$$
R^{n+1}(\lambda, \alpha) \subset R^{n}(\lambda, \alpha) .
$$

Proof. Let $f$ belong to $R^{n+1}(\lambda, \alpha)$ and let it be given by (1.1). Then from (1.5), we have

$$
\operatorname{Re}\left(1+\frac{1}{2(1-\alpha)} \sum_{k=2}^{\infty} k[1+(k-1) \lambda]^{n+1} a_{k} z^{k-1}\right)>\frac{1}{2}
$$

Now

$$
\begin{aligned}
\left(D^{n} f(z)\right)^{\prime}= & 1+\sum_{k=2}^{\infty} k[1+(k-1) \lambda]^{n} a_{k} z^{k-1} \\
= & \left(1+\frac{1}{2(1-\alpha)} \sum_{k=2}^{\infty} k[1+(k-1) \lambda]^{n+1} a_{k} z^{k-1}\right) \\
& *\left(1+2(1-\alpha) \sum_{k=2}^{\infty} \frac{z^{k-1}}{1+(k-1) \lambda}\right) .
\end{aligned}
$$

Applying Lemma 2.2, with $c_{0}=1$ and $c_{k}=1 /(1+k \lambda), k=1,2, \ldots$, we get

$$
\operatorname{Re}\left(1+2(1-\alpha) \sum_{k=2}^{\infty} \frac{z^{k-1}}{[1+(k-1) \lambda]}\right)>\alpha .
$$

Applying Lemma 2.1 to $\left(D^{n} f(z)\right)^{\prime}$, we get the required result. 
We also have a better result than Theorem 2.3.

THEOREM 2.4. Let $f \in R^{n+1}(\lambda, \alpha)$. Then $f \in R^{n}(\lambda, \beta)$, where

$$
\beta=\frac{2 \lambda^{2}+(1+3 \lambda) \alpha}{(1+\lambda)(1+2 \lambda)} \geqslant \alpha
$$

Proof. Let $f \in R^{n+1}(\lambda, \alpha)$. It is shown in [9], as an example, that if $\lambda \geqslant 0$ and

$$
g(z)=z+\sum_{k=2}^{\infty} \frac{z^{k}}{1+(k-1) \lambda}
$$

then

$$
\operatorname{Re} \frac{g(z)}{z}>\frac{4 \lambda^{2}+3 \lambda+1}{2(1+\lambda)(1+2 \lambda)}
$$

Hence

$$
\operatorname{Re}\left(1+2(1-\alpha) \sum_{k=2}^{\infty} \frac{z^{k-1}}{1+(k-1) \lambda}\right)>\frac{2 \lambda^{2}+(1+3 \lambda) \alpha}{(1+\lambda)(1+2 \lambda)}
$$

Now an application of Lemma 2.1 to $\left(D^{n} f(z)\right)^{\prime}$ in the previous theorem completes the proof.

REMARK 2.5. If we put $n=1$ in Theorem 2.4 , then we have

$$
\operatorname{Re}\left(f^{\prime}(z)+\lambda z f^{\prime \prime}(z)\right)>\alpha \Longrightarrow \operatorname{Re} f^{\prime}(z)>\frac{2 \lambda^{2}+(1+3 \lambda) \alpha}{(1+\lambda)(1+2 \lambda)}
$$

which is an improvement of the result of Saitoh [7] for $\lambda \geq 1$, where he shows that, for $\lambda>0$,

$$
\operatorname{Re}\left(f^{\prime}(z)+\lambda z f^{\prime \prime}(z)\right)>\alpha \Longrightarrow \operatorname{Re} f^{\prime}(z)>\frac{2 \alpha+\lambda}{2+\lambda}
$$

Using Theorem $2.4((n-m)$ times $)$ we get, after some calculations, the following theorem.

THEOREM 2.6. Let $f \in R^{n}(\lambda, \alpha)$ and let $n>m \geqslant 0$. Then $f \in R^{m}(\lambda, \beta)$ if

$$
\beta=\left[\left(\frac{1+3 \lambda}{(1+\lambda)(1+2 \lambda)}\right)^{n-m} \alpha+\frac{2 \lambda^{2}}{(1+\lambda)(1+2 \lambda)} \sum_{k=0}^{n-m-1}\left(\frac{1+3 \lambda}{(1+\lambda)(1+2 \lambda)}\right)^{k}\right] \geqslant \alpha
$$

If we put $m=0$ in Theorem 2.6, we obtain the following interesting result.

COROLlaRY 2.7. Let $f \in R^{n}(\lambda, \alpha)$. Then $\operatorname{Re} f^{\prime}(z)>\beta$, where $\beta$ is given by (2.12) with $m=0$. 
REMARK 2.8. Since $D_{\lambda}$ (given by (1.3)) is a linear function of $\lambda$, it is clear that

$$
R^{n}(\lambda, \alpha) \subset R^{n}\left(\lambda^{\prime}, \alpha\right),
$$

where $\lambda>\lambda^{\prime}$.

The following theorem deals with the partial sum of the functions in $R^{n}(\lambda, \alpha)$. For the proof we need the following result, due to Ahuja and Jahangiri [2].

LEMMA 2.9. Let $-1<t \leqslant S=4.567802$. Then

$$
\operatorname{Re}\left(\sum_{k=2}^{m} \frac{z^{k-1}}{k+t-1}\right)>-\frac{1}{1+t}, \quad z \in \Delta .
$$

THEOREM 2.10. Let $S_{m}(z, f)$ denote the $m$ th partial sum of a function $f$ in $R^{n}(\lambda, \alpha)$. If $f \in R^{n}(\lambda, \alpha)$ and $\lambda \geq 1 / s=0.21892$, then $S_{m}(z, f) \in R^{n-1}(\lambda, \beta)$, where

$$
\beta=\frac{2 \alpha+\lambda-1}{\lambda+1}
$$

Proof. Let $f \in R^{n}(\lambda, \alpha)$ and let it be given by (1.1). Then from (1.5) we have

$$
\operatorname{Re}\left(1+\sum_{k=2}^{\infty} k[1+(k-1) \lambda]^{n} a_{k} z^{k-1}\right)>\alpha
$$

or

$$
\operatorname{Re}\left(1+\frac{2}{\lambda+1} \sum_{k=2}^{\infty} k[1+(k-1) \lambda]^{n} a_{k} z^{k-1}\right)>\frac{2 \alpha+\lambda-1}{\lambda+1} .
$$

Now

$$
\begin{aligned}
\left(D^{n-1} S_{m}(z, f)\right)^{\prime}= & 1+\sum_{k=2}^{m} k[1+(k-1) \lambda]^{n-1} a_{k} z^{k-1} \\
= & \left(1+\frac{2}{\lambda+1} \sum_{k=2}^{\infty} k[1+(k-1) \lambda]^{n} a_{k} z^{k-1}\right) \\
& *\left(1+\frac{\lambda+1}{2 \lambda} \sum_{k=2}^{m} \frac{z^{k-1}}{1 / \lambda+(k-1)}\right), \quad \lambda>0
\end{aligned}
$$

From Lemma 2.9, we see that, for $\lambda \geq 1 / s=0.21892$,

$$
\operatorname{Re} \sum_{k=2}^{m} \frac{z^{k-1}}{1 / \lambda+(k-1)}>-\frac{\lambda}{\lambda+1},
$$

hence

$$
\operatorname{Re}\left(1+\frac{\lambda+1}{2 \lambda} \sum_{k=2}^{m} \frac{z^{k-1}}{1 / \lambda+(k-1)}\right)>\frac{1}{2},
$$

and the result follows by application of Lemma 2.1. 
Now we prove the following theorem.

THEOREM 2.11. The set $R^{n}(\lambda, \alpha)$ is convex.

Proof. Let the functions

$$
f_{i}(z)=z+\sum_{k=2}^{\infty} a_{k i} z^{k} \quad(i=1,2)
$$

be in the class $R^{n}(\lambda, \alpha)$. It is sufficient to show that the function $h(z)=\mu_{1} f_{1}(z)+$ $\mu_{2} f_{2}(z)$, with $\mu_{1}$ and $\mu_{2}$ nonnegative and $\mu_{1}+\mu_{2}=1$, is in the class $R^{n}(\lambda, \alpha)$.

Since

$$
h(z)=z+\sum_{k=2}^{\infty}\left(\mu_{1} a_{k 1}+\mu_{2} a_{k 2}\right) z^{k}
$$

then from (2.4) we have

$$
\left(D^{n} h(z)\right)^{\prime}=1+\sum_{k=2}^{\infty} k\left(\mu_{1} a_{k 1}+\mu_{2} a_{k 2}\right)[1+(k-1) \lambda]^{n} z^{k-1},
$$

hence

$$
\begin{aligned}
\operatorname{Re}\left(D^{n} h(z)\right)^{\prime}= & \operatorname{Re}\left(1+\mu_{1} \sum_{k=2}^{\infty} k[1+(k-1) \lambda]^{n} a_{k 1} z^{k-1}\right) \\
& +\operatorname{Re}\left(1+\mu_{2} \sum_{k=2}^{\infty} k[1+(k-1) \lambda]^{n} a_{k 2} z^{k-1}\right) .
\end{aligned}
$$

Since $f_{1}, f_{2} \in R^{n}(\lambda, \alpha)$, this implies that

$$
\operatorname{Re}\left(1+\mu_{i} \sum_{k=2}^{\infty} k[1+(k-1) \lambda]^{n} a_{k i} z^{k-1}\right)>1+\mu_{i}(\alpha-1) \quad(i=1,2) .
$$

Using (2.25) in (2.24), we obtain

$$
\operatorname{Re}\left(D^{n} h(z)\right)^{\prime}>1+\alpha\left(\mu_{1}+\mu_{2}\right)-\left(\mu_{1}+\mu_{2}\right),
$$

and since $\mu_{1}+\mu_{2}=1$, the theorem is proved.

Hallenbeck [4] showed that

$$
\operatorname{Re} f^{\prime}(z)>\alpha \Rightarrow \operatorname{Re} \frac{f(z)}{z}>(2 \alpha-1)+2(1-\alpha) \log 2 .
$$

Using Theorem 2.3 and (2.27), we obtain the following theorem.

THEOREM 2.12. Let $f \in R^{n}(\lambda, \alpha)$. Then

$$
\operatorname{Re} \frac{D^{n} f(z)}{z}>(2 \alpha-1)+2(1-\alpha) \log 2
$$

This result is sharp as can be seen by the function $f_{x}$ given by (3.1). 
3. Extreme points. The extreme points of the closed convex hull of $R(\alpha)$ were determined by Hallenbeck [4]. We denote the closed convex hull of a family $F$ by clco $F$, and we make use of some results in [4] to determine the extreme points of $R^{n}(\lambda, \alpha)$.

THEOREM 3.1. The extreme points of $R^{n}(\lambda, \alpha)$ are

$$
f_{x}(z)=z+2(1-\alpha) \sum_{k=2}^{\infty} \frac{x^{k-1} z^{k}}{k[1+(k-1) \lambda]^{n}}, \quad|x|=1, z \in \Delta .
$$

Proof. Since $D^{n}: f \rightarrow D^{n} f$ is an isomorphism from $R^{n}(\lambda, \alpha)$ to $R(\alpha)$, it preserves the extreme points and, in [4], it is shown that the extreme points of $R(\alpha)$ are

$$
z+2(1-\alpha) \sum_{k=2}^{\infty} \frac{1}{k} x^{k-1} z^{k}, \quad|x|=1, z \in \Delta .
$$

Hence from (1.5), we see that the extreme points of $\operatorname{clco} R^{n}(\lambda, \alpha)$ are given by (3.1). Since the family $R^{n}(\lambda, \alpha)$ is convex (Theorem 2.6) and therefore equal to its convex hull, we get the required result.

As consequences of Theorem 3.1, we have the following corollary.

COROLlary 3.2. Let $f$ belong to $R^{n}(\lambda, \alpha)$ and let it be given by (1.1). Then

$$
\left|a_{k}\right| \leqslant \frac{2(1-\alpha)}{k[1+(k-1) \lambda]^{n}}, \quad k \geqslant 2
$$

This result is sharp as shown by the function $f_{x}(z)$ given by (3.1).

COROLlARY 3.3. If $f \in R^{n}(\lambda, \alpha)$, then

$$
\begin{array}{ll}
|f(z)| \leqslant r+\sum_{k=2}^{\infty} \frac{2(1-\alpha)}{k[1+(k-1) \lambda]^{n}} r^{k}, & |z|=r, \\
\left|f^{\prime}(z)\right| \leqslant 1+\sum_{k=2}^{\infty} \frac{2(1-\alpha)}{[1+(k-1) \lambda]^{n}} r^{k-1}, & |z|=r .
\end{array}
$$

This result is sharp as shown by the function $f_{x}(z)$ given by (3.1) at $z=\bar{x} r$.

4. Convolution properties. Ruscheweyh and Sheil-Small [6] verified the PolyaSchoenberg conjecture and its analogous results, namely, $C * C \subset C, C * S^{\star} \subset S^{\star}$, and $C * K \subset K$, where $C, S^{\star}$, and $K$ denote the classes of convex, starlike, and close-toconvex univalent functions, respectively. In the following, we prove the analogue of the Polya-Schoenberg conjecture for the class $R^{n}(\lambda, \alpha)$.

THEOREM 4.1. Let $f \in R^{n}(\lambda, \alpha)$ and $g \in C$. Then $f * g \in R^{n}(\lambda, \alpha)$.

Proof. It is known that if $g$ is convex univalent in $\Delta$, then

$$
\operatorname{Re} \frac{g(z)}{z}>\frac{1}{2}
$$


Using convolution properties, we have

$$
\operatorname{Re}\left(D^{n}(f * g)(z)\right)^{\prime}=\operatorname{Re}\left(\left(D^{n} f(z)\right)^{\prime} * \frac{g(z)}{z}\right),
$$

and the result follows by application of Lemma 2.1.

THEOREM 4.2. Let $f$ and $g$ belong to $R^{n}(\lambda, \alpha)$. Then $f * g \in R^{n}(\lambda, \beta)$, where

$$
\beta=\frac{\lambda(2 \alpha+1)+4 \alpha-1}{2(\lambda+1)} \geqslant \alpha
$$

Proof. Let $g(z)=z+\sum_{k=2}^{\infty} b_{k} z^{k} \in R^{n}(\lambda, \alpha)$, then

$$
\operatorname{Re}\left(1+\sum_{k=2}^{\infty} k[1+(k-1) \lambda]^{n} b_{k} z^{k-1}\right)>\alpha .
$$

Let $c_{0}=1$ and

$$
c_{k}=\frac{\lambda+1}{(k+1)[1+k \lambda]^{n}}, \quad k \geqslant 1
$$

Then $\left\{c_{k}\right\}_{k=0}^{\infty}$ is a convex null sequence. Hence, by Lemma 2.2, we have

$$
\operatorname{Re}\left(1+\sum_{k=2}^{\infty} \frac{\lambda+1}{k[1+(k-1) \lambda]^{n}} z^{k-1}\right)>\frac{1}{2} .
$$

Now we take the convolution of (4.4) and (4.6) and apply Lemma 2.1 to obtain

$$
\operatorname{Re}\left(1+(\lambda+1) \sum_{k=2}^{\infty} b_{k} z^{k-1}\right)>\alpha
$$

or

$$
\operatorname{Re} \frac{g(z)}{z}=\operatorname{Re}\left(1+\sum_{k=2}^{\infty} b_{k} z^{k-1}\right)>\frac{\lambda+\alpha}{\lambda+1} .
$$

Hence

$$
\operatorname{Re}\left(\frac{g(z)}{z}-\frac{2 \alpha+\lambda-1}{2(\lambda+1)}\right)>\frac{1}{2}
$$

Since $f \in R^{n}(\lambda, \alpha)$, by applying Lemma 2.1 , we obtain

$$
\operatorname{Re}\left(\left(D^{n} f(z)\right)^{\prime} *\left(\frac{g(z)}{z}-\frac{2 \alpha+\lambda-1}{2(\lambda+1)}\right)\right)>\alpha
$$

or

$$
\operatorname{Re}\left(\left(D^{n} f(z)\right)^{\prime} * \frac{g(z)}{z}\right)>\frac{\lambda(2 \alpha+1)+4 \alpha-1}{2(\lambda+1)}=\beta,
$$

and by (4.2), the result follows. 
REMARK 4.3. If we put $\lambda=0$ in Theorem 4.2, we get the corresponding result for functions in $R(\alpha)$, given by Ahuja [1].

\section{REFERENCES}

[1] O. P. Ahuja, Univalent functions whose derivatives have a positive real part, Rend. Mat. (7) 2 (1982), no. 1, 173-187.

[2] O. P. Ahuja and M. Jahangiri, On the derivatives of a family of analytic functions, Math. Japon. 47 (1998), no. 1, 67-72.

[3] L. Fejér, Über die positivität von summen, die nach trigonometrischen oder Legendreschen funktionen fortschreiten. I, Acta Szeged 2 (1925), 75-86 (German).

[4] D. J. Hallenbeck, Convex hulls and extreme points of some families of univalent functions, Trans. Amer. Math. Soc. 192 (1974), 285-292.

[5] S. Ponnusamy, Differential subordination and starlike functions, Complex Variables Theory Appl. 19 (1992), no. 3, 185-194.

[6] St. Ruscheweyh and T. Sheil-Small, Hadamard products of Schlicht functions and the PólyaSchoenberg conjecture, Comment. Math. Helv. 48 (1973), 119-135.

[7] H. Saitoh, Properties of certain analytic functions, Proc. Japan Acad. Ser. A Math. Sci. 65 (1989), no. 5, 131-134.

[8] G. Ş. Sălăgean, Subclasses of univalent functions, Complex Analysis-Fifth Romanian-Finnish Seminar, Part 1 (Bucharest, 1981), Lecture Notes in Math., vol. 1013, Springer, Berlin, 1983, pp. 362-372.

[9] Z. Zhongzhu and S. Owa, Convolution properties of a class of bounded analytic functions, Bull. Austral. Math. Soc. 45 (1992), no. 1, 9-23.

F. M. Al-Oboudi: Mathematics Department, Science Sections, Girls College of Education, Sitteen Street, Malaz, Riyadh 11417, Saudi Arabia

E-mail address: ryte1mi@gcpa.edu.sa 


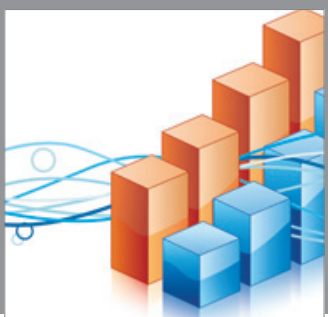

Advances in

Operations Research

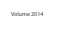

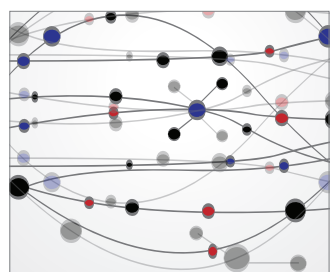

\section{The Scientific} World Journal
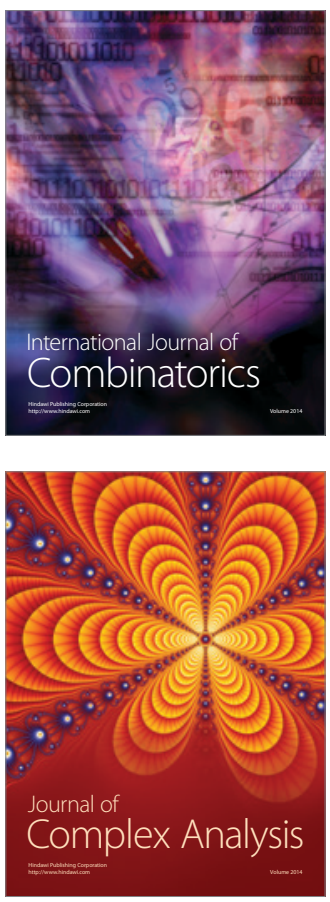

International Journal of

Mathematics and

Mathematical

Sciences
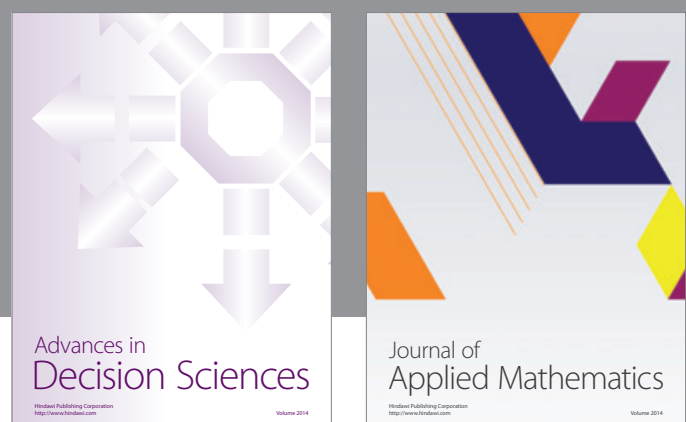

Journal of

Applied Mathematics
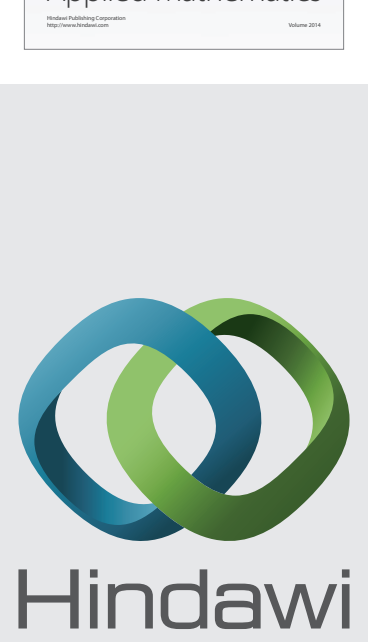

Submit your manuscripts at http://www.hindawi.com
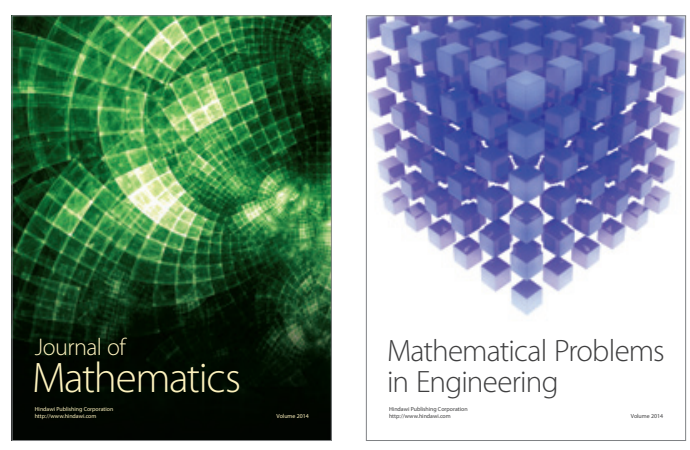

Mathematical Problems in Engineering
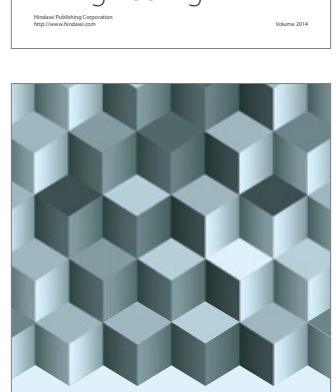

Journal of

Function Spaces
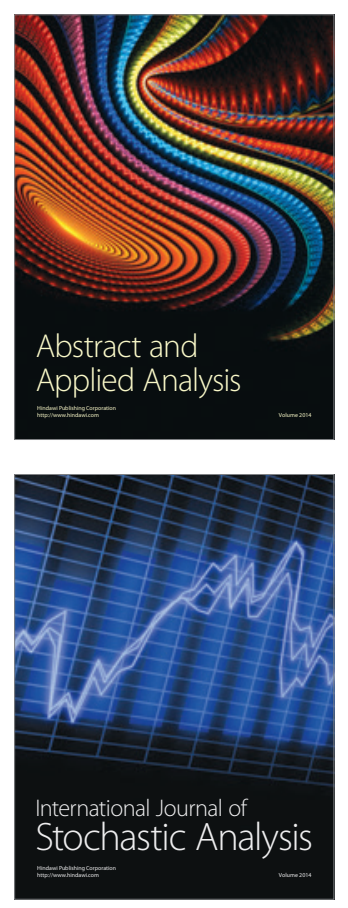

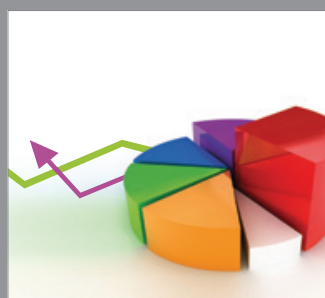

ournal of

Probability and Statistics

Promensencen
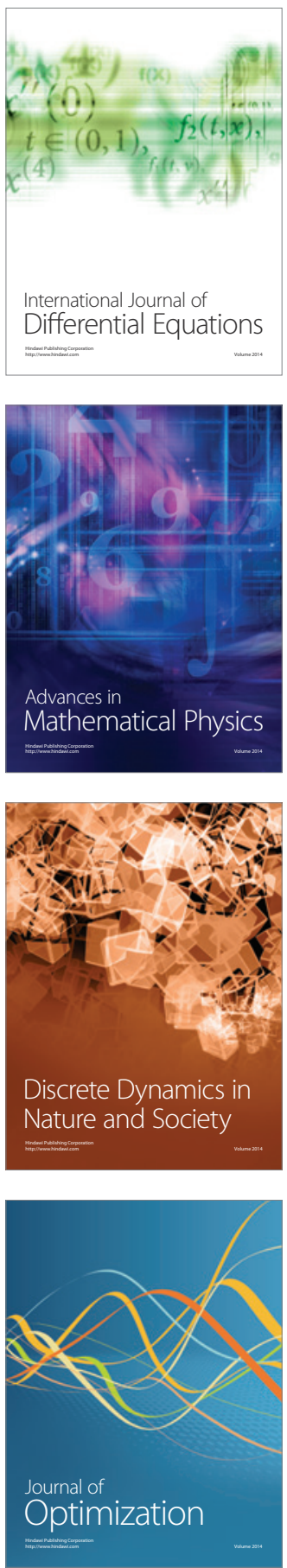